\title{
Robotically Assisted Intraoperative Ultrasound with Application to Ablative Therapy of Liver Cancer
}

\author{
Emad M. Boctor ${ }^{\mathrm{a}}$, Russell H. Taylor ${ }^{\mathrm{a}}$, Gabor Fichtinger ${ }^{\mathrm{a}}$, Michael A. Choti ${ }^{\mathrm{b}}$ \\ ${ }^{a}$ Center of Computer-Integrated Surgical Systems and Technologies, ${ }^{b}$ Dept. of Surgery and \\ Oncology, Johns Hopkins University
}

\begin{abstract}
Management of primary and metastatic tumors of the liver remains a significant challenge to the health care community worldwide. There has been an increasing interest in minimally invasive ablative approaches that typically require precise placement of the tissue ablator within the volumetric center of the tumor, in order to achieve adequate destruction. Standard clinical technique involves free hand transcutaneous ultrasonography (TCUS) in conjunction with manual positioning of the tissue ablator. Unfortunately, existing TCUS systems suffer from many limitations. TCUS fails to identify nearly half of all treatable liver lesions, whereas intraoperative or laparoscopic US provides excellent tissue differentiation. Furthermore, freehand manipulation of the US probe critically lacks the level of control, accuracy, and stability required for guiding liver ablation. Volumetric reconstruction from sparse and irregular 2D image data is suboptimal. Variable pressure from the sonographer's hand also causes anatomic deformation. Finally, maintaining optimal scanning position with respect to the target lesion is crucial, but virtually impossible to achieve with freehand guidance. In response to these limitations, we propose the use of a fully encoded dexterous robotic arm to manipulate the US probe during surgery.
\end{abstract}

Keywords: Calibration, 3D Ultrasound, Medical Robotics, Liver Surgery, RF Ablation, Registration

\section{INTRODUCTION}

Hepatocellular carcinoma is one of the most common malignancies, accounting for more than 1 million cases per year ${ }^{1}$. Metastatic disease to the liver, primarily from colorectal cancer is the most frequent hepatic malignancy in the United States. The principal potentially curative treatment for these patients is liver resection, resulting in 5-year survival rates between 25 and $55 \%$. Unfortunately, most patients with primary and secondary liver cancer are not candidates for resection, primarily due to tumor location or underlying liver disease. For these reasons, an increasing interest has been focused on interstitial ablative approaches for the treatment of unresectable liver tumors. In addition to increasing the number of patients eligible for curative therapy of liver cancer, Local tissue ablation is performed with lower morbidity than resection and can be employed as a minimally invasive approach, including percutaneously and laparoscopically. Studies have demonstrated the efficacy of interstitial ablative approaches for the treatment of hepatic tumors, including chemical ablation, cryoablation, and thermal ablation using energy sources like radiofrequency, laser, microwave, or focused ultrasound. Thermal ablation using radiofrequency energy is the most frequently used modality ${ }^{2,3}$ (Figure 1), but other techniques are also used, including ethanol injection, cryotherapy ${ }^{4}$, microwave ${ }^{5}$, and interstitial photon radiation. ${ }^{6}$

Despite advances in the effectiveness the end-effectors, precise placement of the ablator device and monitoring of the zone of necrosis are still unsolved problems. The precise location of hepatic tumors is usually determined by a preoperative imaging modality such as computed tomography (CT) or magnetic resonance imaging (MRI). However, the preoperative planning information can't be used actively or solely during surgery to guide resections or probe placements for ablative therapies. On the other hand, moving these scanners (CT, MRI) to the surgery room would help actively in the intraoperative guidance. However, these scanners (CT, MRI) suffer from the following problems. First, 
these systems are expensive, based on the unit cost, installation cost, cost per operation and cost of compatibility (i.e. MRI should be placed in a magnetic free interference environment). Second, MRI and CT are large equipment and this occupied volume could make standard operations more difficult. Third, these imaging devices are not real-time, and depending on actual system architecture, their latencies may be quite significant.

Intraoperative ultrasonography (IOUS) may be the ideal imaging modality, because it provides excellent anatomical imaging, while also real-time, interactive, and is widely popular in diagnostic and therapeutic procedures ${ }^{7}$. Thanks to its specificity and sensitivity, it is of primary importance in disorders of the pancreas, biliary tree, and liver ${ }^{2}$, especially in early stages of the disease. The diagnostic accuracy of alternative techniques, i.e. pre-operative imaging (angiography, Scintigraphy, CT, ultrasonography), and surgical exploration, doesn't exceed 60-80\%. ${ }^{8}$ Intra-operative ultrasonography, however, allows for early diagnosis and precise localization of many diseases, and it is an excellent guidance tool for accurate and radical surgical treatment.

In current surgical practice, intraoperative ultrasound guided liver ablation is performed mainly in two major steps. First, tumors are identified by preoperative imaging primarily CT and MR. Second, these tumors are operatively (or laparoscopically) localized by intraoperative ultrasonography (IOUS). Current methodology requires manual IOUS in conjunction with free hand positioning of the ablation probe under ultrasound guidance (Figure 1). Simultaneous manual handling of the B-mode ultrasound (US) probe and the ablator device is a challenging task that is prone to significant errors in the hands of even the most experienced physicians. ${ }^{9}$ IOUS imaging provides perhaps the optimal and most unavailable method for targeting, but its current two-dimensional format leads to significant interuser variation in results. Tissue deformation and target motion make it extremely difficult to place the ablator device precisely into the target. Irregularly shaped target volumes typically require multiple insertions and several overlapping thermal lesions, which are even more challenging to accomplish in a precise and timely manner without causing excessive damage to surrounding normal tissues. In answer to these problems, we propose to integrate robot-assisted three-dimensional intraoperative ultrasound (3D-IOUS) with a passive lightweight arm to facilitate precise placement of a thermal ablator into the liver and to monitor the progress of tissue ablation with real-time ultrasound (Figure 2).

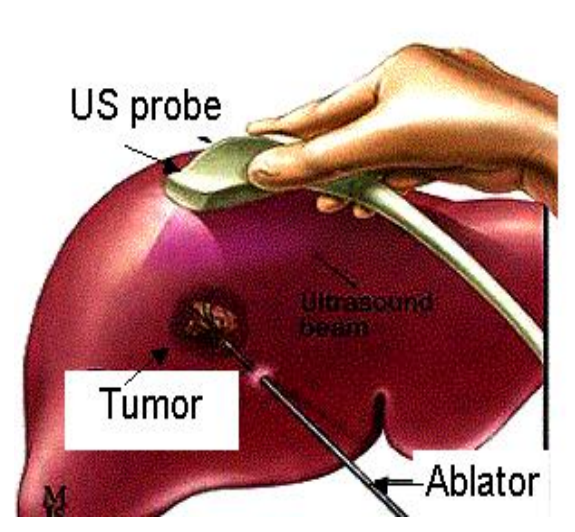

Figure 1: Radiofrequency ablation of liver tumor

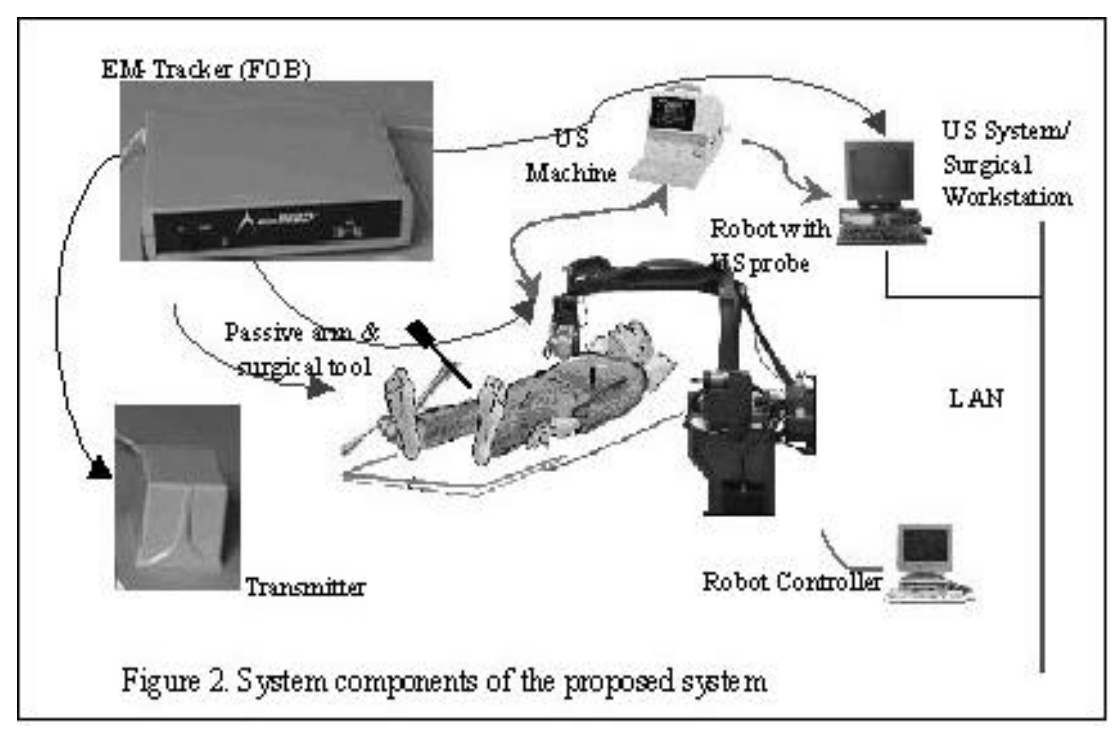


Regardless to whether the ablation is delivered in transcutaneous, laparoscopic, or open surgical setting, the process always involves moving the tip of the device to a pre-selected entry point, then entering the device into the tissue till it reaches a pre-selected target point, and only then the actual tissue ablation can take place. Realtime ultrasound imaging has active role throughout the entire procedure, hence precise, predictable, and repeatable control of the US probe is critically needed. A robot-assisted ultrasound system would provide many benefits: enabling more structured and optimized 3D ultrasound datasets, providing consistent reliable guidance to interventions, help registering multimodality images, and avoiding fatigue and occasional musculoskeletal injury of the sonographer ${ }^{10}$. In liver ablation surgery, robot-assisted ultrasound can maintain the pressure profile induced by the US probe, thus minimize tissue deformation and motion artifacts. Telesurgery and teleradiology are also potential beneficiaries of robotic ultrasound.

Liver ablation is an ideal setting for the invocation of guided ultrasound technologies. Improved real-time guidance for planning, delivering and monitoring the ablative therapy could provide the missing tool needed to allow accurate and effective application of this promising therapy.

The hypothesis of our research program is that the integration of robot-assisted ultrasound with spatial co-registration of the ablator device can ultimately make these procedures more accurate, faster, effective, and less expensive in the long run. Robotic assistance may also reduce the dependency of outcome manual skill of the surgeon, leaving actual medical knowledge and experience the decisive factor of the surgery. The actual system embodiment of this paradigm features robotic 3D ultrasound, RF ablator attached to a passive arm of six degrees-of-freedom (DOF), both spatially coregistsred by a real-time tracking device, under the control of a central planning and monitoring computer.

\section{MATERIALS AND METHODS}

\subsection{System overview}

The key feature in our prototype system is real-time spatial tracking of both the ultrasound probe and RF ablator. This scenario applies to all forms of ultrasonography: transcutaneous, laparoscopic, and intraoperative, of which we explore the latter one in this project. Figure 3 illustrates the four possible alternatives for the intraoperative US guided liver ablation, depending on how we manipulate the ablator and ultrasound transducer with respect to one another. The first alternative is to have a "passive-passive" system where the ablator is guided by a passive arm and the US transducer is used in freehand scanning. ${ }^{9}$ In a "passive-active" system, an active arm guides the ablator while the ultrasound transducer is still held manually. In an "active-active" system both the ablator and US probe are under robot control. The subject of this paper is the fourth variant, the "active-passive" system, where the US probe is guided and controlled by a robotic arm while the ablator is attached to a passive articulated mechanical linkage.

The actual system embodiment that is featured in Figure 2 is composed of: (1) LARS (Laparoscopic Assistance Robotic System) robot developed previously by Taylor et al. ${ }^{11}$, (2) a commercial ultrasound scanner (Aloka SSD1400, Japan), (3) a pulsed magnetic field position and orientation measurement system (Flock of Birds, model 6D FOB, Ascension Technology Corp., Burlington, VT, USA), (4) an radiofrequency (RF) ablation probe (XL, RITA Medical Inc.) supported by a passive arm; and (5) a central workstation to provide overall control, data management, and graphical user interface. The hub of the system is the central workstation that provides capturing ultrasound images, recording data from the FOB tracker, building the 3D US volume, and planning and monitoring of the delivered treatment. The particular US probe used in this study is a $3.5 \mathrm{MHz}$ curved array transducer from Aloka. A real-time image capture board (Matrox, MeteorII) is installed in the central computer to acquire the US images through the video output interface. 


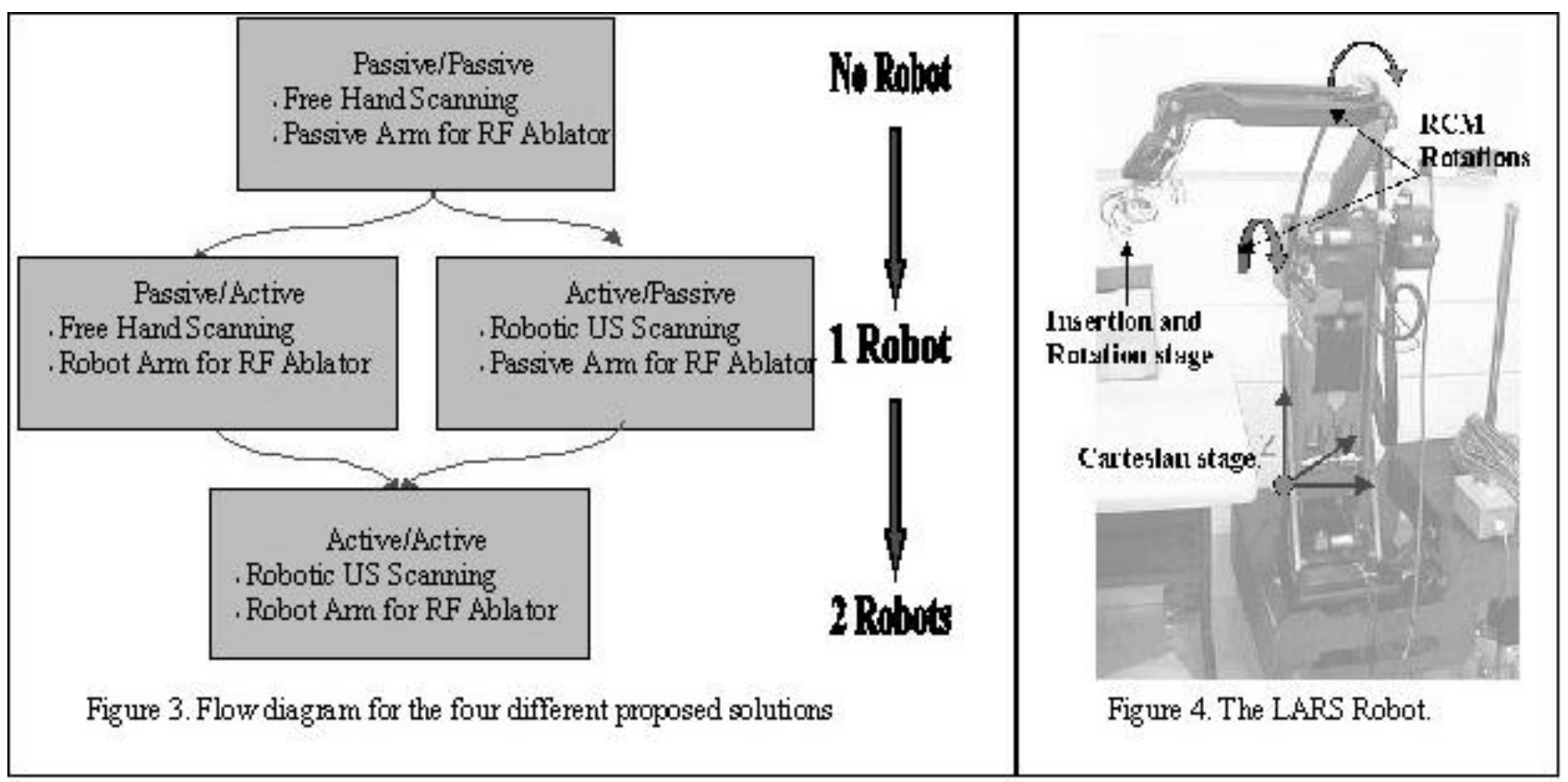

\subsection{Robotic system (LARS)}

Taylor was the first to propose remote center of motion (RCM) concept ${ }^{11}$, which denotes the ability to provide angular orientation about a fixed point in space. Taylor's invention has become the foundation for many modern laparoscopic and needle placement robots known today, including such well-known systems as the DaVinci (by Intuitive Surgical Systems). The LARS robot, Laparoscopic Assistance Robotic System, is a kinematically redundant manipulator composed of a proximal translation component, along with a distal remote center of motion component that provides three rotations and one controlled insertion motion that passes through the RCM point. The LARS, as shown in Figure 4, consists of three-axis linear Cartesian motion stage, a two-axis parallel four bar linkage providing two rotations $\left(\mathrm{R}_{\mathrm{x}}\right.$ and $R_{y}$ ) about the remote center of motion, and a two-axis distal component providing an insertion motion, $s$, and rotation $R_{s}$ about the instrument axis, which passes through the remote center of motion.

A unit called RCC (Robot Control Computer) controls the LARS and essentially acts as a server. A dedicated computer runs the robot server and it is accessed by a client application running on the central planning/monitoring workstation. The communication takes place via TCP-IP connection over a local network. The client application is sealed off from the robot server; they communicate with the use of a small library of functions, such as InitializeRobot, MoveRotationalStage, and so forth. Procedure, error, and status codes are also communicated to the client application. The robot software infrastructure has been has been developed at our Center, under the name of Modular Robot Control (MRC) library. The MRC library is a generalized set of portable $\mathrm{C}++$ classes for distributed and modular robot control; it covers forward and inverse kinematics, joint level control, force sensors and peripheral support.

In this project, we have adopted the LARS robot to acquire 3D US data in preliminary studies for our minimally invasive robot-assisted liver ablation research program. In order to achieve the coupling between the US and the LARS, the following steps had to be completed: (1) Calibrate a 6-DOF force sensor resident on the LARS and integrate it with the MRC library. (2) Design and build end-effectors to hold the US probe in the RCM point. (3) Integrate the LARS in the 3D US software. The integration of LARS required the implementation of several image acquisition scenarios. One of these scenarios is to move the robot in a steady way, covering the volume of interest and in the same time capture dynamically the US images along with their tracker readings. Another way is to move the robot incrementally to capture statically the US images and the tracker readings. The steadiness of the robot is one of the prominent features, allowing 
for static tracking. In general static tracking is more accurate than dynamic tracking in freehand tracked ultrasound, because here one does not have to calibrate the system for the delay between the image and the tracker reading. ${ }^{12}$

The force sensor is incorporated in the system to serve two purposes. First, the imaging protocol starts with an exploration phase, when the user guides the arm around the volume of interest. This is accomplished by using the multidimensional force-sensor that interprets the intentions of the user and allows the robot to comply and learn this path. The robot can thus acquire a 3D US volume by capturing position/force tagged 2D US images according to a predefined scanning profile. Second, maintaining the same pressure profile allows accurate repeat scans. This helps not only better management of data gaps but also produce high quality and reproducible volume reconstruction. Once the volume is available, we can plan the treatment and execute it with the use of the passive arm, monitored in repeat scans performed the robot-assisted ultrasound.

\subsection{Tracked RF ablator and passive arm}

Radiofrequency ablation is one of the most promising minimally invasive techniques (Figure 1) for the treatment of unresectable hepatic tumors. ${ }^{1}$ Recent studies have reported favorable survival rates and excellent rates of local control after such ablation, especially in patients with hepatocellular carcinoma. ${ }^{2,3}$ So, it is utmost important to identify the location of the ablator tool before insertion and plan for a highly accurate and minimum invasive targeting. Because ultrasound imaging cannot show the trajectory of the needle before it is inserted, simultaneous spatial tracking of both the US probe and needle is the only option to know where the needle is oriented with respect to the target. Therefore, spatial tracking is a fundamentally important aspect of the proposed system.

Tracking the ablator device and identify its end points with respect to the tracker frame (FOB) is called calibration of the $\mathrm{RF}$ ablator. The location and orientation $\left(\mathrm{F}_{\text {sensor }}\right)$ of the FOB's sensor with respect to the base frame or FOB's transmitter $\left(\mathrm{F}_{\text {transmitter }}\right)$ are captured real-time. In order to get the unknown position vectors for the two end points, a Pivot Calibration method was used as shown in Figure 6.

In addition, the process of ablation though one entry point recalls the need of pivoting support. For these reasons, the RF probe is supported by an adjustable rigid mechanical structure comprising the following components (Figure 5): (1) A 6DOF passive arm provides moving the needle to the point of insertion. (2) A 5-bar remote center of motion (RCM) linkage, attached to the passive arm, provides two independent rotations for the needle to pivot around the point of insertion. (3) A passive un-encoded linear stage holds the ablator needle and provides insertion into the liver. (4) Friction brakes hold the rotation and linear motion stages in place. (5) A Lego attachment holds interchangeably the Flock of Bird and Polaris sensors. (6) A plastic extension for the RF probe that allows for accurate pivoting calibration around both ends of the needle.

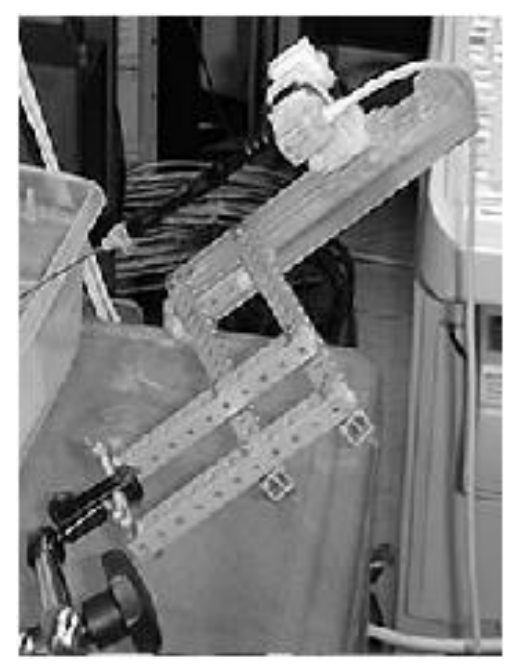

Figure 5: Tracked passive arm

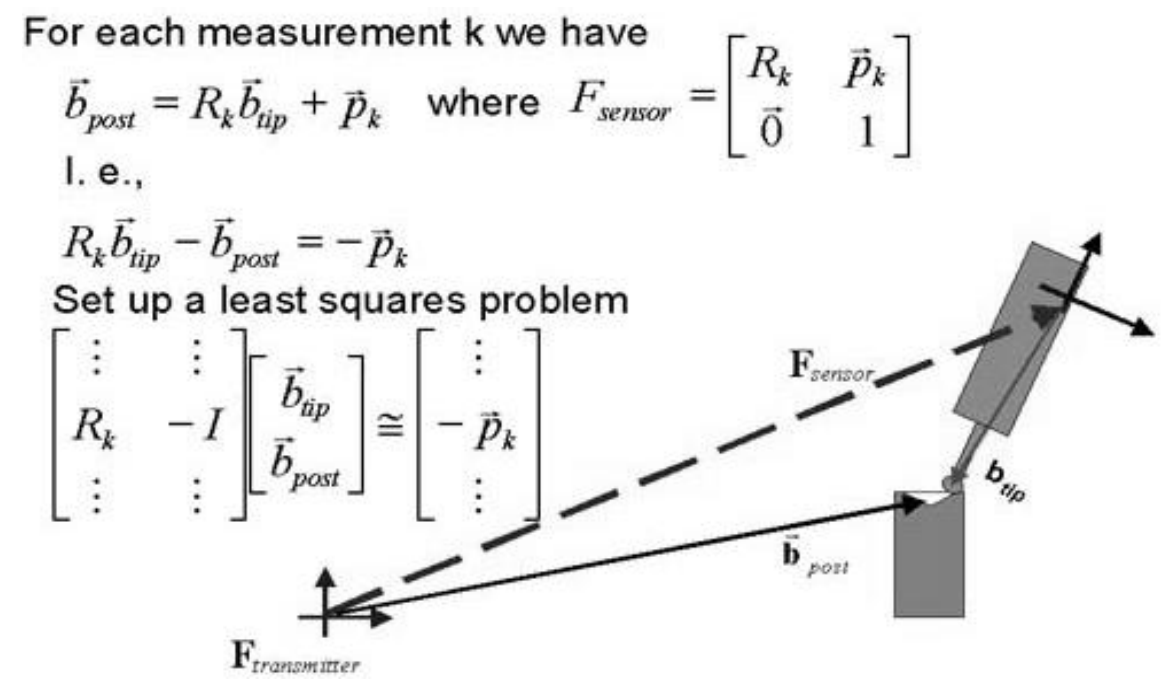

Figure 6: Pivot calibration method to define the 2 end points of the RF ablator in the tracker coordinate system. 


\subsection{Tracked robotic 3D ultrasound system}

Figure 7 depicts the basic elements of any 3D US system based on 2D US scanner ${ }^{13}$, critically involving tracking and scanning methods. Tracking could be accomplished using mechanical, electromagnetic, or optical devices; scanning methods can be either manual or robotic.

The following modules represent the main components of any tracked 3DUS system:

- Acquisition and Synchronization Module: Capturing the two streams of US data and tracker information in a synchronized way.

- Reconstruction Module: Converting the scattered arrays of images into a structured 3D voxel array using an appropriate reconstruction and compounding algorithm.

- Processing and Visualization: Enhancing the US images by applying smoothing and/or gap filling algorithms for better visualization.

- Calibration Module: Calibrating the US beam, i.e. to determining the transformation between the US image space and the tracker space.

The overall accuracy of reconstruction in a freehand 3D US system is dependent, to a certain extent, on the following factors. First, good synchronization (temporal calibration or accurate tagging between the captured image and the tracker reading) is required to overcome the uncertainties of dynamic tracking and the unknown internal delays of the tracking device (which in the order of 20-80 msec). Second, optimal spatial reconstruction and to some extent, motion artifact compensation is required. This is a significant challenge, considering the sparse and irregular 2D image data, variable pressure from the sonographer's hand causing variable deformation of the imaged tissues. Third, preprocessing and gap filling algorithms are required because there is no fixed gap pattern for every freehand scan.

Robotically assisted 3D US system, however, overcomes all above challenges of the freehand system. In a robotic US system, there is no need for synchronization to tag the captured US image with the tracker reading, since scanning is performed in a well-controlled incremental fashion. In other words, the robot can be gated to start the next movement only after capturing the US image and reading the tracker are completed. In addition, the robotic system provides more favorable management of data gaps, which in turn leads to faster and more efficient data preprocessing.

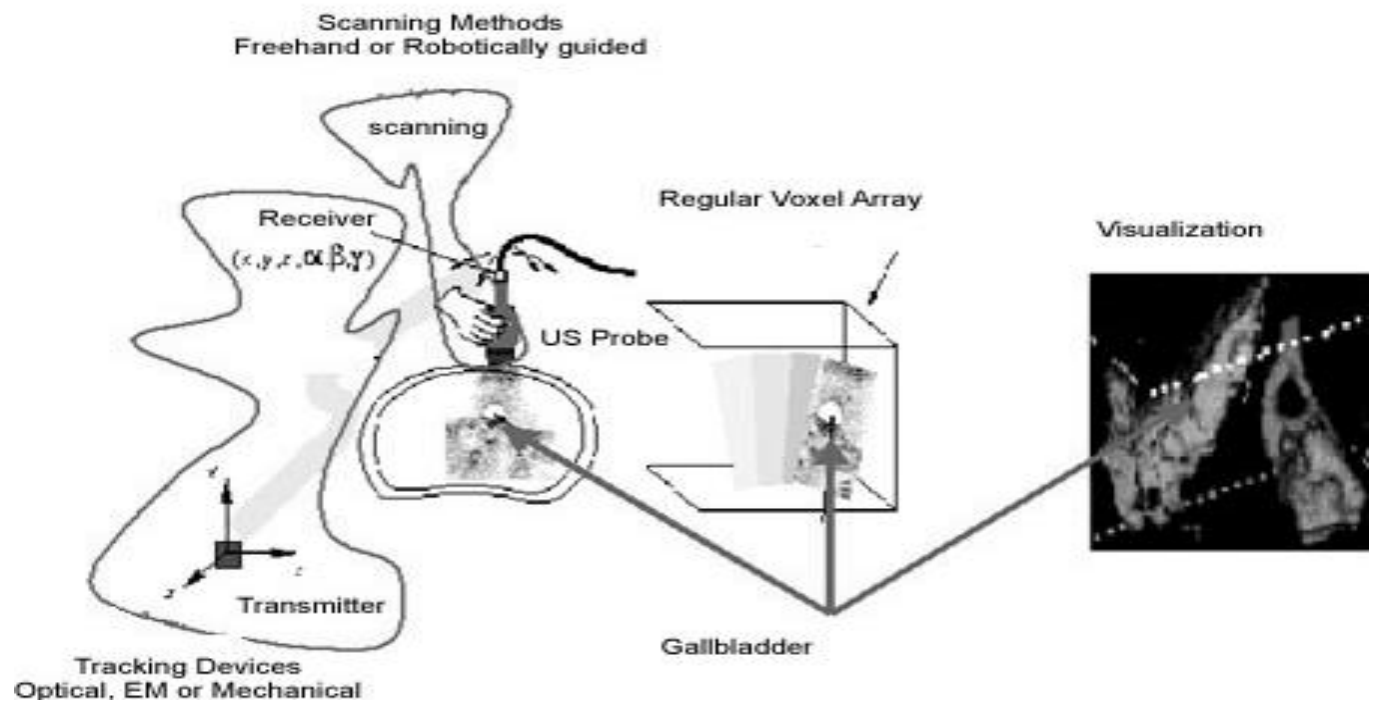

Figure 7. Generic Tracked US System 
Altogether, the robotic US system has substantial improvements over the freehand US system, in terms of data acquisition, synchronization, reconstruction, and preprocessing. At the same time, calibration is critically important to the accuracy of the 3D ultrasound system.

\subsection{Calibration}

The spatial calibration process involves determining the 6-DOF rigid-body transformation between the position sensor and the corner of the B-scan plane, and also the $x$ and $y$ dimensions of the pixels (typically in $\mathrm{mm} / \mathrm{pixel}$ units). Spatial calibration is usually performed by scanning and reconstructing some known object and then, using the discrepancy between the reconstructed shape and the known shape, re-estimating the calibration parameters. Knowing this transformation matrix, one can place every pixel in the image coordinate system into the reconstruction space. Our 3DUS system uses a novel calibration technique discussed separately in a companion paper ${ }^{14}$, presenting a comparative study between the known calibration methods in literature ${ }^{15}$ (Cross-wire, Three-wires and Single-wall methods). This work led to a new calibration method and phantom (Figure 8,9). This new method (Hopkins method) yielded significant improvements in reconstruction and calibration accuracy. In summary, the Hopkins phantom, in conjunction with a multisided tank, gives us the following advantages:

- The number of necessary images is reduced by a factor of 20 (conservatively)

- The numbers of data points are increased in each image

- Avoiding the "beam width" problem, which is a constant problem with the Single-wall phantom

- Easy to construct, without special design requirements for the given US probe

- The clarity of features in the ultrasound images facilitates full automation of the calibration process

- Images can be collected in a wide range of scanning motion

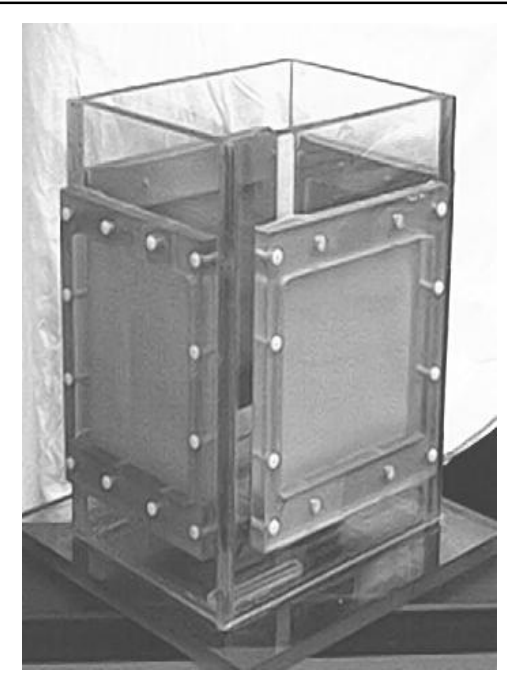

Figure 8: The Multisided tank is shown with five sides (top and four rubber windows). At the bottom, there is a piece of Lego to attach different phantoms.

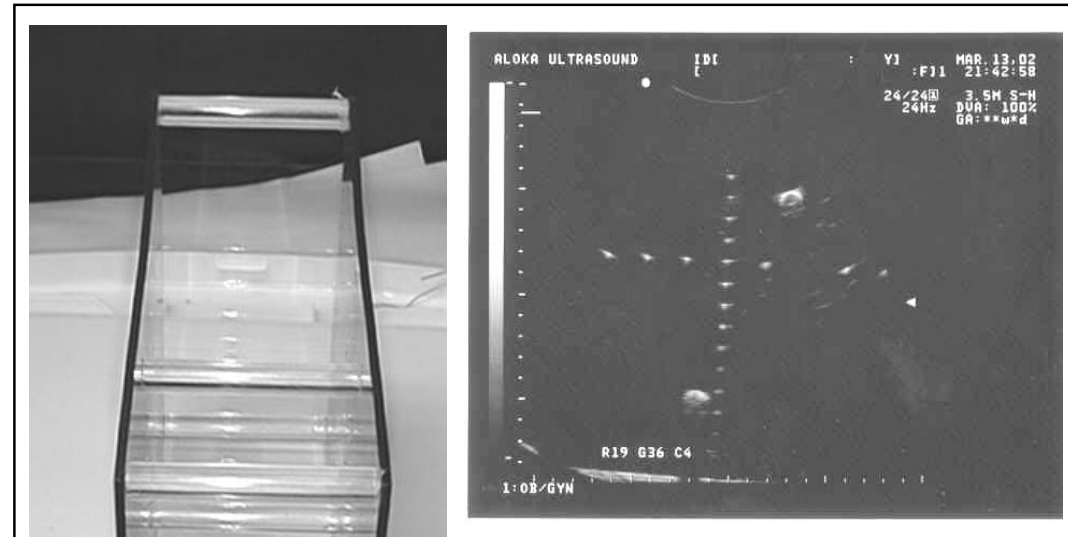

Figure 9: The Hopkins phantom (left) and a typical image of it (right), illustrating the large number of data points that can be collected, in a potentially automated manner. 


\subsection{Planning and visualization software}

For graphical user interface, we adapted the 3D Slicer medical data visualization package ${ }^{16}$. This is a public domain open source system primarily developed by the Surgical Planning Laboratory at the Brigham and Women Hospital (http://www.slicer.org), with sustained contribution from our group. The main strength that we added to Slicer is a generic 3D US processing module, providing synchronized real-time capture of 2D US data and position information, and robust assembly of a spatially registered 3D volume from sparse and irregular 2D information. The software also provides a customizable real-time overlay display of the US volume, RF ablator tip with its current ablating range, and the current 2D ultrasound slice, as shown in Figure 11. Tumor coverage can be planned with single-spot and overlapping multiple ablations. Beside the aforementioned visual overlay, are other visual and metric tools help the user in placing the ablator. The system reposts the current insertion depth that can be double-checked against the ruler on the passive unencoded insertion stage, it shows the distance between the actual and planned RF ablator, and also provides coordinate systems and landmarks to ease navigation in the virtual space.

\section{EXPERIMENTS AND RESULTS}

In testing and evaluating this system, we have adopted this procedure by testing first the stand-alone accuracy of US calibration, RF ablator tool calibration, robotic 3D US reconstruction appearance compared to freehad system, and finally testing the system integration.

Through series of US spatial calibration experiments based on Cross-wire and Hopkins phantoms, it has been proved that Hopkins calibration can produce higher reconstruction accuracy, about 30\% improvements over the Cross-wire calibration with the same settings (Figure 10). In addition, an average image of the Hopkins phantom yields 20 data points. This means that a highly reasonable set of 220 points could be collected from just 10-12 images, which is sufficient to produce a very accurate calibration matrix, if the images are collected from all sides of the tank. Furthermore, the optimization framework remains stable with even fewer images and data points. For example, the condition number of the Jacobian matrix in Levenberg-Marquardt method near to solution remains bounded at about 150 while using only 80 points (3-4 images) collected from all side combined (Figure 11). Most importantly, average reconstruction precision for Hopkins phantom is about $0.6 \mathrm{~mm}$ with a standard deviation of $0.28 \mathrm{~mm}$ and maximum deviation of $2.4 \mathrm{~mm}$. These numbers represents the deviation and uncertainty in placing US pixels in space.

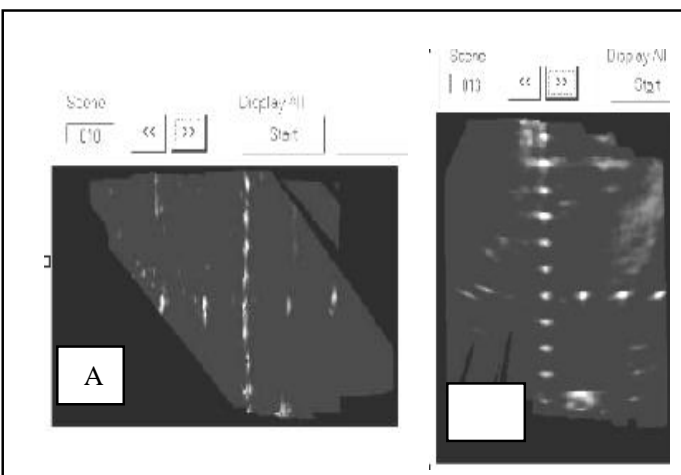

Figure 10: "A" shows the reconstruction of the Hopkins phantom by using the calibration matrix of the Cross-wire method. "B" shows the reconstruction based on Hopkins calibration parameters. It is obvious that Hopkins method leads to higher reconstruction accuracy.

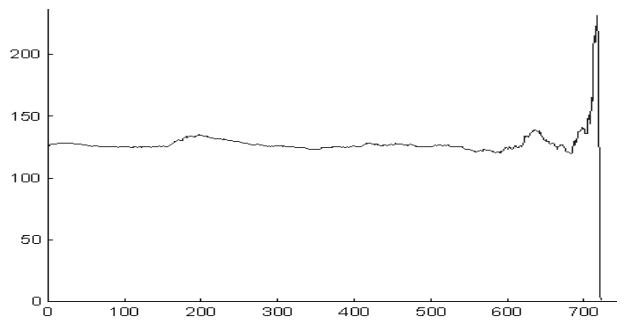

Figure 11: The vertical-axis is the condition number while the horizontal-axis is the number of points dropped from the optimization (total is 700 in this case). It shows that the condition number remains stable to the 600 level, which means $80-100$ points is enough to make the system well conditioned. 

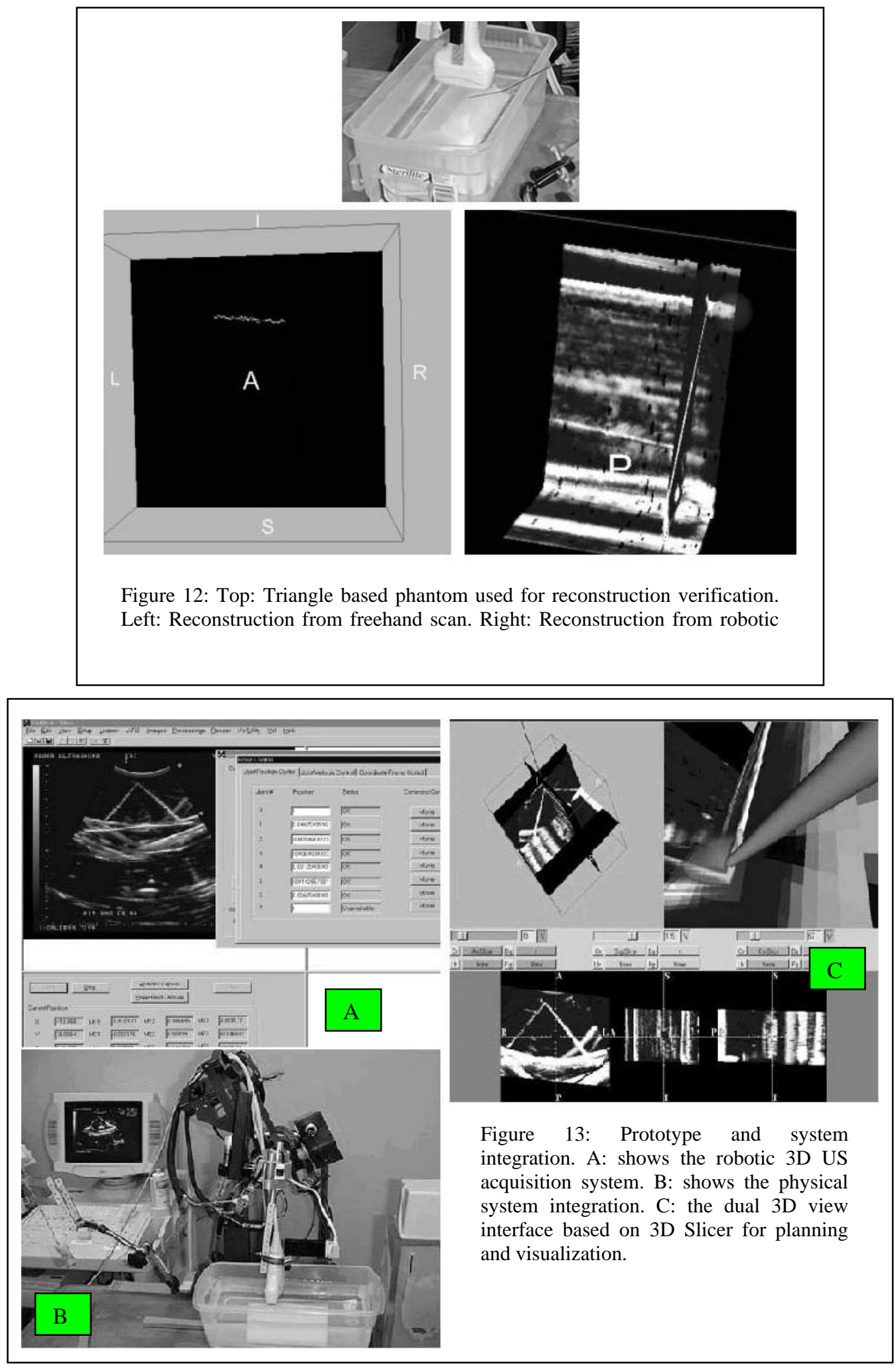
We have conducted a comparative in-vitro experiments between the robotic ultrasound system and the freehand 3D system. The robotic system provided better quality and accuracy of the 3D reconstruction as shown in figure 12 . This is afforded by regular step scanning, better management of the predefined gap distribution, and provision for static tracking.

In the presented phase of the project, we concentrated on the overall mechanical accuracy of the system, assuming stationary target in an ideal setup. Figure 13-A shows a snapshot of the robotic US S/W with real time US capturing, tracking information and robot control parameters. Figure13-B is a typical scene of the integrated system during one of the testing experiments using the triangle-shaped phantom shown in top panel of Figure 12. 3D Slicer visualization software for planning, simulating and supervision the ablation with dual 3D views appears on figure 13-C. Results from these experiments show the ability of the method to accurately reach a target. Both 2D and 3D US guiding schemes proved to be able to hit the target (verified by visual and US confirmation); however ablation planning based on single 2D US was not always sufficient in covering the target volume, which was always achieved with 3D US planning. The method of verification was visual observation in the tank under water, on graphical display, and in live 2D US. The average targeting error was under $1.5 \mathrm{~mm}$, and all accuracy readings were consistently in the order of the tracking device itself (FOB, Flock of Bird by Ascension Tech., Inc.). Much of the uncertainty can be attributed to the calibration errors of RF ablator tool. The standard deviation in repeated pivot calibrations was between 1.0 and $2.0 \mathrm{~mm}$, the variability is probably due to the flexibility of the needle rim.

\section{CONCLUSION}

This project demonstrates that RF ablation of liver tumors may benefit from the use of robotically assisted 3D ultrasonography. We have proven that this system is more favorable in many respects than tracked freehand 3D US. Our results are rather preliminary. We have solved the problem of image deformation caused by the variable pressure profile, but still must extend the work to account for respiratory motion artifacts.

While we are proceeding with improving on the manipulation of the ultrasound probe, significant efforts are also devoted to perfecting our ablator placement. Technology. To reduce targeting time and enhance accuracy, we are also experimenting with motorized rotation and needle insertion stages, previously developed at the Johns Hopkins University Urobotics $\mathrm{Lab}^{17}$. Simultaneous spinning and advancing the needle could reduce tissue resistance and subsequently reduce deformation and displacement of the target tissue. This, however, requires more sophisticated needle drivers, currently under development at the Johns Hopkins University.

In the presented phase of the project, we concentrated on the overall mechanical accuracy of the system, assuming stationary target in an ideal setup. As these results were most satisfactory, we move toward more realistic setups. In the near future, we will be preparing for ex-vivo on animal models, and eventually human cadaver tests, in order to address uncertainties in real-time tracking and deformation and mechanical motion artifacts. Next, we will perform in-vivo animal tests to account for respiratory motion artifacts and other in-vivo uncertainties, in realistic operating room setup.

\section{ACKNOWLEDGEMENTS}

The authors gratefully acknowledge the support and help offered by Mr. John Walsh (ALOKA Inc.) by providing the SSD1400 US equipment. We also thank Ankur Kapoor, Ameet Jain and Anton Deguet for their input and assistance. This work is supported by the National Science Foundation under Engineering Research Center grant \#EEC 9731478.

\section{REFERENCES}

1. Nakakura EK, Choti MA. Management of hepatocellular carcinoma. Oncology 2000;14(7):1085-98; discussion 1098-102.

2. Choti MA. Surgical management of hepatocellular carcinoma: resection and ablation. J Vasc Interv Radiol. 2002 Sep;13(9 Suppl):S197-203. 
3. Choti, MA. "Hepatic radiofrequency ablation". The Cancer Journal 2000; 6(4): 291-2.

4. Kuszyk BS, Boitnott JK, Choti MA, Bluemke DA, Sheth S, Magee CA, Horton KM, Eng J, Fishman EK. Local tumor recurrence following hepatic cryoablation: radiologic-histopathologic correlation in a rabbit model. Radiology. 2000 Nov;217(2):477-86.

5. Izumi N, Asahina Y, Noguchi O, Uchihara M, Kanazawa N, Itakura J, Himeno Y, Miyake S, Sakai T, Enomoto N: Risk factors for distant recurrence of hepatocellular carcinoma in the liver after complete coagulation by microwave or radiofrequency ablation. Cancer. 2001 Mar 1;91(5):949-56.

6. Koniaris LG, Chan DY, Magee C, Solomon SB, Anderson JH, Smith DO, De Weese T, Kavoussi LR, Choti MA. Focal hepatic ablation using interstitial photon radiation energy. J Am Coll Surg. 2000 Aug;191(2):164-74.

7. Angelini L. and Caratozzolo M. Intraoperative echography: the state of the art. Ann Ital Chir, 70(2):223-30, MarchApril 1999.

8. Russo A., La Rosa C., Cajozzo M., Spallitta I., Demma I., Modica G., and Bazan P. Screening for liver metastases of colorectal carcinoma by the routine use of intraoperative echography. Minerva Chir, 17(44):1893-900, 1989.

9. Emad Boctor, G. Fichtinger, R. H. Taylor, M. A. Choti, "Tracked 3D ultrasound in radio-frequency liver ablation", SPIE Medical imaging2003, San Diego 15-20 Feb.

10. H.E. Vanderpool, E.A. Friis, B.S. Smith, and K.L. Harms, "Prevalence of carpal tunnel syndrome and other workrelated musculoskeletal problems in cardiac sonographers," Journal of Occupational Medicine, vol. 35, pp. 604-610, June 1993.

11. Taylor RH, Funda J, Eldridge B, Gruben K, LaRose D, Gomory S, Talamini M, Kavoussi LA, and Anderson JH: A Telerobotic Assistant for Laparoscopic Surgery. IEEE EMBS Magazine Special Issue on Robotics in Surgery. 1995. pp. 279-291

12. Fabrice Chassat and Stephane Lavallée, "Experimental Protocol of Accuracy Evaluation of 6-D Localizers for Computer-Integrated Surgery: Application to Four Optical Localizers", MICCAI1998, Springer-Verlag, Berlin, Heidelberg, 1496:277-284.

13. Emad M. Boctor et. al. "PC-Based system for calibration, Reconstruction, Processing and Visualization of 3D Ultrasound Data Based on a Magnetic-Field Position and Orientation Sensing System”, IEEE/ICCS-2001, Lecture Notes in Computer Science, Springer Verlag, 2001.

14. Emad M. Boctor, A. Jain, M. Choti, R. Taylor, and G. Fichtinger, "A Rapid Calibration Method for Registration and 3D Tracking of Ultrasound Images Using Spatial Localizer", SPIE Medical imaging2003, San Diego 15-20 Feb.

15. R.W. Prager, R. Rohling, A. Gee, and Berman L. "Rapid calibration for 3D freehand ultrasound," Ultrasound in Medicine and Biology, vol. 24, no. 6, pp. 855-869, 1998.

16. Gering DT et al. "An integrated visualization system for surgical planning and guidance using image fusion and an open MR”, J Magn Reson Imaging. 2001 Jun;13(6):967-75.

17. Stoianovici D: URobotics - Urology Robotics at Johns Hopkins. Comp Aid Surg, 2001, (6):360-369 